\title{
Hybrid 2D-3D optical devices for integrated optics by direct laser writing
}

\begin{abstract}
Martin Schumann ${ }^{1,2}$, Tiemo Bückmann ${ }^{1}$, Nico Gruhler ${ }^{2}$, Martin Wegener ${ }^{1,2}$ and Wolfram Pernice ${ }^{2}$
Integrated optical chips have already been established for application in optical communication. They also offer interesting future perspectives for integrated quantum optics on a chip. At present, however, they are mostly fabricated using essentially planar fabrication approaches like electron-beam lithography or UV optical lithography. Many further design options would arise if one had complete fabrication freedom in regard to the third dimension normal to the chip without having to give up the virtues and the know-how of existing planar fabrication technologies. As a step in this direction, we here use three-dimensional dip-in direct-laser-writing optical lithography to fabricate three-dimensional polymeric functional devices on pre-fabricated planar optical chips containing $\mathrm{Si}_{3} \mathrm{~N}_{4}$ waveguides as well as grating couplers made by standard electron-beam lithography. The first example is a polymeric dielectric rectangular-shaped waveguide which is connected to $\mathrm{Si}_{3} \mathrm{~N}_{4}$ waveguides and that is adiabatically twisted along its axis to achieve geometrical rotation of linear polarization on the chip. The rotator's broadband performance at around $1550 \mathrm{~nm}$ wavelength is verified by polarization-dependent grating couplers. Such polarization rotation on the optical chip cannot easily be achieved by other means. The second example is a whispering-gallery-mode optical resonator connected to $\mathrm{Si}_{3} \mathrm{~N}_{4}$ waveguides on the chip via polymeric waveguides. By mechanically connecting the latter to the disk, we can control the coupling to the resonator and, at the same time, guarantee mechanical stability of the three-dimensional architecture on the chip.
\end{abstract}

Light: Science \& Applications (2014) 3, e175; doi:10.1038/lsa.2014.56; published online 6 June 2014

Keywords: direct laser writing; integrated optics; nanophotonic circuits; polarization conversion

\section{INTRODUCTION}

Nanophotonic integrated circuits allow for realizing complex optical functionality by assembling many individual devices into fullscale systems. Relying on established fabrication routines originally developed for the realization of integrated electrical circuits, such devices can be manufactured with high accuracy and reproducibility. For application in telecommunication and optical signal processing, this typically requires the use of electron-beam lithography or advanced optical lithography to fabricate structures with deep submicrometer dimensions. To date, the necessary lithographic resolution is readily available for structuring quasiplanar integrated optical components. By combining planar lithography with subsequent transfer into materials with higher refractive index, high-quality optical devices can be realized. These include nanophotonic waveguides with propagation loss down to $0.1 \mathrm{~dB} \mathrm{~cm}{ }^{-1}$, optical resonators with quality factors approaching a billion ${ }^{2,3}$ as well as a rich library of devices for signal processing including interferometers, ${ }^{4-7}$ filters ${ }^{8,9}$ and tunable systems. ${ }^{10-13}$ Fabricated circuits find applications in traditional linear optics, ${ }^{14,15}$ non-linear optics ${ }^{16-18}$ and recently also for the realization of integrated non-classical and quantum-optical circuits. ${ }^{19-21}$

While remarkable progress has been made in developing suitable devices for many applications, the design process is generally borrowed from top-down fabrication and thus, imposes stringent limitations on devices which cannot be easily obtained with planar technology. This includes the realization of three-dimensional (3D) photonic crystals, ${ }^{22,23}$ curved surface optical elements ${ }^{24}$ and lens structures. ${ }^{25-28}$ Similarly, multi-layer topographies ${ }^{29,30}$ as usually employed in electronic chips are difficult to achieve. Even in planar circuits limitations arise, for example, for the realization of polarization control ${ }^{31-36}$ or for the implementation of free-standing structures required for tunable $^{37,38}$ and optomechanical applications. ${ }^{39-41}$ Therefore, advanced lithography techniques that provide access to truly $3 \mathrm{D}$ geometries are of particular interest. Among the available options direct laser writing (DLW $)^{42}$ is especially attractive for the combination with planar circuits because of direct fabrication compatibility. Besides providing full writing flexibility, ${ }^{43-45}$ DLW also offers lithographic resolution better than $100 \mathrm{~nm}^{46}$ and fast writing speed. ${ }^{47}$ Using DLW polymer photonic wirebonds for interchip communication have been demonstrated, ${ }^{48}$ illustrating that waveguiding in DLW-written photonic components can be achieved with low loss. ${ }^{50}$ Here we extend current photonic integrated circuits to full three dimensions by combining planar lithography with DLW. Using DLW allows us to realize circuit elements that cannot be fabricated with traditional methods as a powerful approach for next-generation on-chip optical components.

${ }^{1}$ Institute of Applied Physics and DFG-Center for Functional Nanostructures (CFN), Karlsruhe Institute of Technology (KIT), 76131 Karlsruhe, Germany and ${ }^{2}$ Institute of Nanotechnology (INT), 76344 Eggenstein-Leopoldshafen, Germany

Correspondence: Dr W Pernice, Institute of Nanotechnology (INT), Hermann-von-Helmholtz-Platz 1, 76344 Eggenstein-Leopoldshafen, Germany

E-mail: wolfram.pernice@kit.edu

Received 6 December 2013; revised 21 February 2014; accepted 25 February 2014 


\section{MATERIALS AND METHODS}

\section{Device fabrication}

We first fabricate planar nanophotonic circuits using high-quality silicon wafers thermally oxidized to a thickness of $2.6 \mu \mathrm{m}$. A top layer of $450 \mathrm{~nm}$ stoichiometric silicon nitride is subsequently deposited by low-pressure chemical vapor deposition. The growth conditions are adjusted to lower the internal tensile stress in the nitride film to avoid film cracking after post-deposition cool-down. To structure the nanophotonic circuits, we employ ma-N 2403 negative tone resist for exposure with a Jeol $530050 \mathrm{kV}$ electron-beam lithography system. After exposure, the samples are developed in MF-319 and a reflow procedure is applied to reduce the residual surface roughness of the resist. The samples are placed on a hot-plate at $110{ }^{\circ} \mathrm{C}$ for $1 \mathrm{~min}$, which leads to a partial softening of the ma- $\mathrm{N}$ resist and subsequent smoothing of the as-written resist surface. The pattern is then transferred into the silicon nitride layer using $\mathrm{CHF}_{3} / \mathrm{O}_{2}$ plasma in an Oxford Plasmalab 80 Plus reactive-ion etching system. To realize rib waveguides, the silicon nitride layer is fully etched, so that the resulting nanophotonic components have a thickness of $450 \mathrm{~nm}$.

In a second lithography step, the nanoscribe Photonic Professional $3 \mathrm{D}$ lithography system is employed to write $3 \mathrm{D}$ optical components on the pre-structured integrated optical chips. For mechanical stability, the chip is glued onto a sample holder and then fully covered by the liquid dip-in photoresist (Nanoscribe IP Dip). A computer-controlled sample stage allows for precise manipulation of the chip position. In the dip-in configuration, which we employ for DLW of all our objects, the objective lens of the writing system is immersed in the photoresist. This allows for convenient writing on top of opaque substrates, like the silicon carrier layer we use during our fabrication. With the help of alignment markers written in the first lithography step in the vicinity of the waveguides (Figure 1c), the DLW system is aligned relative to the nanophotonic circuit with submicrometer accuracy. Since the chip plane is generally slightly rotated and tilted with respect to the coordinate system of the sample stage, coordinates have to be transformed before the writing can start. The DLW by itself is carried out by focusing the writing laser into the photoresist. The photoresist is designed such that single photons from the writing laser cannot be absorbed, but a two-photon absorption can induce polymerization. Since nonlinear processes scale with intensity, the polymerization occurs where the intensity is the highest, i.e., in the focal volume of the laser. Furthermore, the polymerization process exhibits a threshold behavior. Thus, only a volume within a certain iso-intensity surface (a 'voxel') given by the threshold intensity of the resist is polymerized. Hence, by moving the sample, arbitrary $3 \mathrm{D}$ trajectories can be polymerized, with the resolution being limited by the voxel size. In our setup, typical voxels are ellipsoids with a lateral diameter of $200 \mathrm{~nm}$ and an axial diameter of $500 \mathrm{~nm}$.

The structures we fabricate are several voxel sizes large in all three dimensions. Therefore, we cannot write the whole 3D device (e.g., a $3 \mathrm{D}$ bridge waveguide) using a single DLW trajectory. Instead, we decompose the volume of the $3 \mathrm{D}$ device to be written into slices less than a voxel size apart from each other. These slices are then filled up using a rectangular spiral pattern. By writing the spiral patterns slice by slice using DLW, we finally obtain the designed 3D structure.

After DLW, the chip is removed from the sample holder and developed with mr-Dev 600 and IPA successively. Typically, we blow-dry the samples using a nitrogen gun. For more delicate structures like disk resonators, however, drying is carried out using a Leica EM CPD030 critical point dryer. Fabricated free-standing DLW structures show good mechanical stability and do not collapse even after
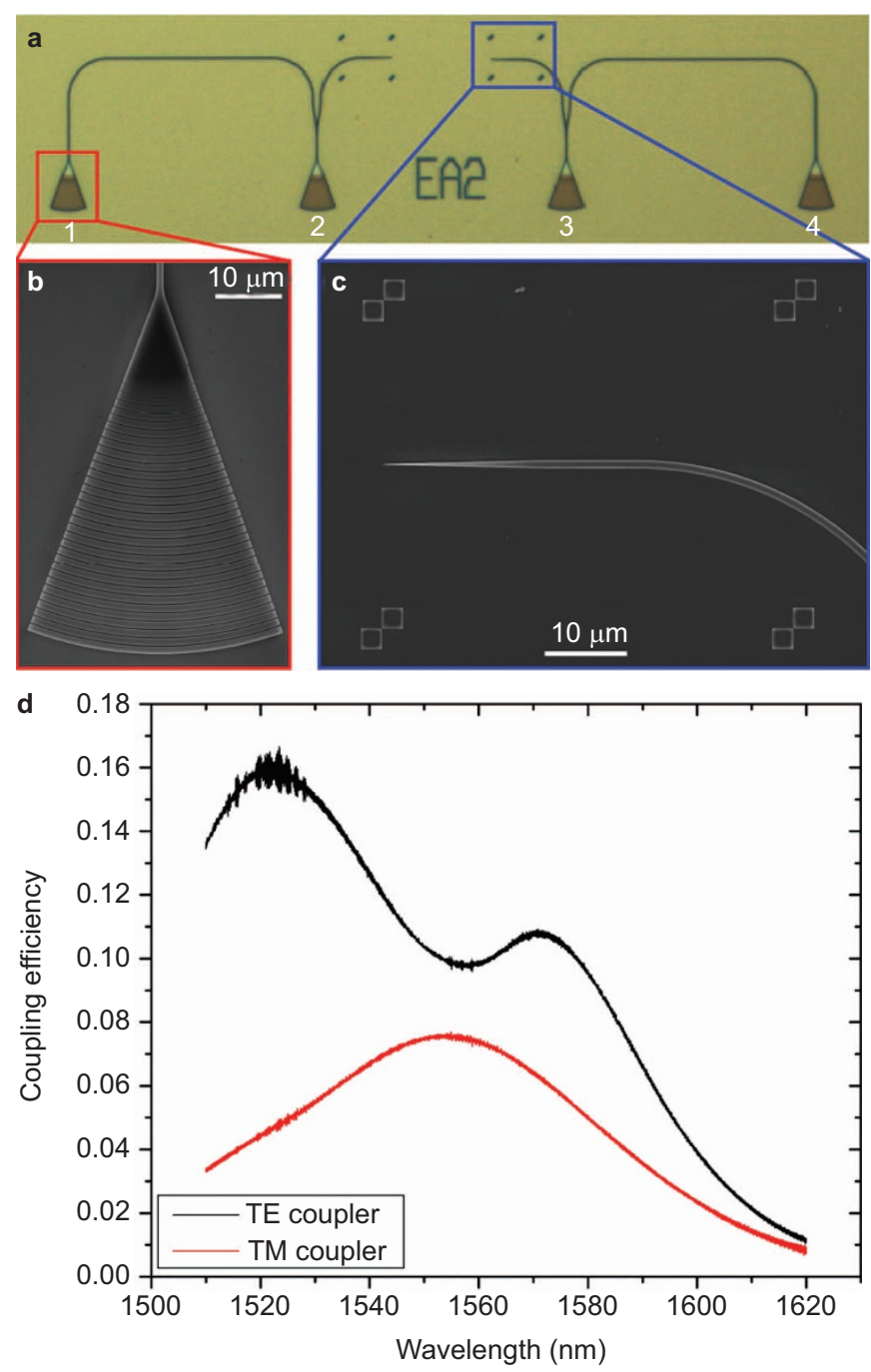

Figure 1 Nanophotonic circuit layout. (a) Optical micrograph of a nanophotonic circuit for the characterization of 3D photonic components, including focusing grating couplers (b: SEM image), Y-splitters and tapered waveguides (c SEM micrograph). The 3D optical component is written into the gap between two facing tapers using DLW. (d) Transmission spectrum of a reference photonic circuit. Two types of grating couplers are employed, where each couples to either TE-like (black curve) or TM-like (red curve) waveguide mode efficiently. DLW, direct laser writing; SEM, scanning electron microscope; TE, transverse electric; $\mathrm{TM}$, transverse magnetic; 3D, three-dimensional.

repeated measurement sessions or after mechanical shock. Using atomic force microscopy, we can determine the surface roughness of the polymer structures after development. From area scans of $0.5 \times 1 \mu \mathrm{m}^{2}$ on several written structures, we find typical root-meansquare roughness of $5 \mathrm{~nm}$, well below the wavelength of the photonic circuits in the telecoms C-band.

\section{Measurement set-up}

In order to characterize the fabricated devices, transmission measurements at near-infrared wavelengths are performed. We use a continuously tunable laser (New Focus TLB-6600) to cover the spectral band between $1510 \mathrm{~nm}$ and $1620 \mathrm{~nm}$, fiber-coupled to single-mode optical fibers. After passing through a fiber polarization rotator for additional polarization control, the laser output is sent to the chip using an optical fiber array. The fiber array comprises several single-mode 
fibers with fixed separation, providing multiple access ports to the integrated optical devices. Light from the fiber array is coupled into the nanophotonic waveguides using focusing grating couplers written in the first lithography step. After passing through the on-chip device, the transmitted optical signal is coupled out again through a second grating coupler and finally is detected by a low-noise photodetector (New Focus 2117). To allow for easy measurement of several on-chip devices and straightforward alignment, the chip is mounted on a computer controlled piezomovable sample stage, allowing us to align the grating couplers with respect to the fiber array with high accuracy.

\section{Chip layout and design}

A typical nanophotonic circuit used to characterize 3D optical components is shown in Figure 1a, including nanophotonic waveguides, beam splitters and optical input/output ports. Light is coupled into the circuit through a central focusing grating coupler (close-up in Figure $1 \mathrm{~b}$ ) on port 2 . Shortly afterward, the propagating mode is split $50: 50$ by a Y-splitter. The light is then guided by nanophotonic waveguides that are $450 \mathrm{~nm}$ thick and $1 \mu \mathrm{m}$ wide. This choice of waveguide geometry supports two guided modes at a wavelength of $1550 \mathrm{~nm}$, one being transverse electric (TE)-like, the other one transverse magnetic (TM)-like. One half of the light is guided to a reference port (1), the other half to a tapered waveguide (close-up in Figure 1c). The light in the tapered waveguide is coupled into a $3 \mathrm{D}$ optical component, which previously was fabricated by DLW such that it closes the gap between the facing tapered waveguides. Eventually, the light is coupled back into the nanophotonic circuit and leaves the chip through another grating coupler (port 3).

The usage of grating couplers to access the chip has several advantages. They allow for semi-automatic, contact-free measuring of a multitude of similar devices in one measurement session. At the same time, reasonably high coupling efficiencies can be achieved. ${ }^{50,51}$ Also, grating couplers are mode-sensitive because their efficiency depends on the effective grating index. ${ }^{52}$ Therefore either of the TE or TM modes can be selectively excited using a suitably designed input coupler. Finally, the maxima of the coupling efficiency can easily be shifted spectrally by adjusting the grating period. ${ }^{41}$ Exploiting the latter two aspects, we employ two types of grating couplers that couple the incident light to either the TE-like or the TM-like waveguide mode more efficiently in the investigated wavelength regime. Using a suitably adjusted grating profile, only one mode is coupled in, while the other polarization is suppressed by more than $10 \mathrm{~dB}$ per grating coupler over a large portion of the spectral band covered by our experimental set-up. Exemplary coupling efficiency curves for both TE and TM grating couplers are shown in Figure 1d, optimized for a central coupling wavelength in the telecoms C-band.

\section{RESULTS AND DISCUSSION}

\section{D bridge waveguides}

In order to transfer light from planar waveguides to arbitrary $3 \mathrm{D}$ components, efficient coupling between the nanophotonic circuit and a $3 \mathrm{D}$ waveguide is crucial. Low insertion loss can be achieved by employing inverted tapers which are conveniently used for coupling nanophotonic waveguides to optical fibers in order to overcome the large insertion loss due to modal size mismatch. Such an approach is ideally suited for interconnecting planar and 3D waveguides written by DLW. Therefore, the transmission characteristics of a 3D bridge waveguide are studied.

To achieve efficient mode matching between the planar silicon nitride waveguide and the polymer waveguides written by DLW, we employ an inverse tapering section in the transition region. The silicon nitride waveguide is tapered over a length of $20 \mu \mathrm{m}$ to a width of $50 \mathrm{~nm}$, limited by the resolution of the electron-beam writer. The silicon nitride taper section is covered with a polymer tapered waveguide of larger cross-section. Using finite-difference time-domain (FDTD) simulations ${ }^{53}$ with the software package meep, ${ }^{54}$ we optimize the geometry of the transition region. For the final design of the polymer structure, we use a tapering length of $30 \mu \mathrm{m}$ from a starting width of $3 \mu \mathrm{m}$ to the final width of $4 \mu \mathrm{m}$. The waveguide height is tapered from $5 \mu \mathrm{m}$ to $6 \mu \mathrm{m}$ over the same length. From the FDTD simulation results, we estimate an insertion loss of less than $1 \mathrm{~dB}$ for wavelengths up to $2 \mu \mathrm{m}$. We note, however, that the FDTD simulations do not take the surface roughness of the polymer waveguides into account. Therefore, the simulated propagation loss provides a lower bound for the insertion loss considering only optical losses due to scattering or radiation coupling into the substrate. In the following, the transmission loss induced by a 3D polymer waveguide is considered to be only due to insertion loss at the taper facets.

To experimentally test the simulation results, we employ a nanophotonic device similar to the one shown in Figure 1a, but without port 4. The polymer waveguide is written on our DLW set-up in approximately $40 \mathrm{~min}$, depending on the actual length of the polymer structure. To characterize the properties of the polymer waveguide, we measure transmission through the polymer section from one silicon nitride input waveguide to a second silicon nitride output waveguide. The $3 \mathrm{D}$ bridge waveguide sits in the gap between the planar waveguides leading to ports 2 and 3 , respectively. All three grating couplers are of the same type, i.e., they excite the same waveguide mode when illuminated. In this fashion, the transmission coefficient of the $3 \mathrm{D}$ bridge waveguide can be deduced separately for TE and TM light. For the measurement, we input the laser light on port 2 and detect the signal on port 1 and port 3. Prior to measuring, we optimize the signal on port 3 by aligning the chip relative to the fiber array and adjusting the input polarization such that it fits the preferred polarization of the respective grating coupler. When analyzing the detector signal on port 3 , propagation losses in the silicon nitride waveguides can be neglected. From calibration measurements on purely planar silicon nitride reference circuits, we estimate propagation loss of $0.3 \mathrm{~dB} \mathrm{~cm}^{-1}$. Thus, for the waveguides in this paper (which are only a few hundred micrometers long), the planar propagation loss can be safely neglected. Hence, the signal on port 3 normalized to the laser output power becomes

$$
T_{2 \rightarrow 3}=1 / 2 \alpha^{2} t^{2}
$$

where $\alpha$ is the coupling efficiency of a grating coupler and $t$ is the insertion loss per facet of the bridge waveguide. The factor $1 / 2$ is due to the Y-splitter. As the transmission measured on port 1 is

$$
T_{2 \rightarrow 1}=1 / 2 \alpha^{2}
$$

the insertion loss per facet $t$ is then readily deduced by measuring $T_{2 \rightarrow 1}$ and $T_{2 \rightarrow 3}$ :

$$
t=\sqrt{\frac{T_{2 \rightarrow 3}}{T_{2 \rightarrow 1}}}
$$

For reference purposes, we first analyze polymer waveguides that span the distance between the silicon nitride tapers in contact with the underlying substrate. The fabricated waveguide is $160 \mu \mathrm{m}$ long, including two tapered input sections. For this configuration, we measure a transmission loss of $2 \mathrm{~dB}$, translating into an insertion loss of 
$1 \mathrm{~dB}$ per taper, in fair agreement with the numerical predictions $(0.3 \mathrm{~dB}$ per taper section in the wavelength range between $1500 \mathrm{~nm}$ and $1600 \mathrm{~nm}$ ). However, as mentioned above, in the simulation results, the propagation loss due to surface roughness is not included, thus accounting for the difference. Furthermore, the transmission loss remains below $3 \mathrm{~dB}$ over the entire wavelength range accessible to our measurement set-up (i.e., $100 \mathrm{~nm}$ ) and is thus suitable for broadband operation.

We then perform characterization of fully-3D polymer waveguides by fabricating microbridges between the nitride waveguide sections. To reduce propagation losses due to bending and scattering, the transition from the planar polymer waveguide to the free-standing waveguide sections is achieved with a smooth transition function, which depends on the separation between the waveguides and the elevation
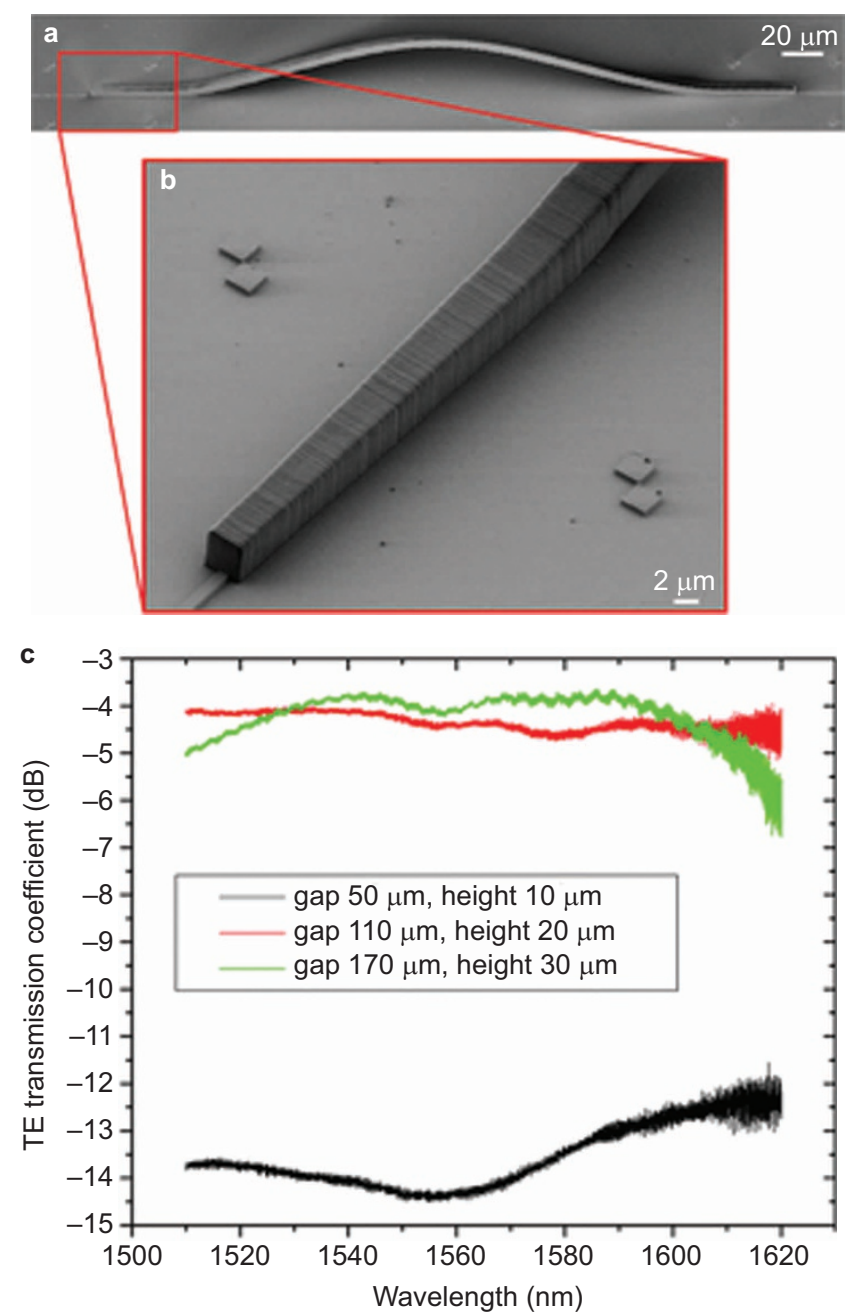

Figure 2 Hybrid planar-3D nanophotonic waveguides. (a) SEM image of a 3D bridge connecting two tapered waveguides. The bridge waveguide is tapered itself, from an initial cross-section of $3 \times 5 \mu \mathrm{m}^{2}$ to a $4 \times 6 \mu \mathrm{m}^{2}$ cross-section (width $\times$ height, design values) in the free-standing region. (b) SEM image of the tapered section of the polymer waveguide. Wall roughness is attributed to the DLW strategy, where individual slices of the 3D bridge are written successively. (c) Measured insertion loss per 3D bridge facet for the TE-like waveguide mode. Longer waveguides show reduced transmission loss because of more adiabatic coupling to the planar silicon nitride waveguides. DLW, direct laser writing; SEM, scanning electron microscope; TE, transverse electric; 3D, threedimensional. of the bridge above the substrate. This measurement is performed for several combinations of length and central height of the 3D bridges. The results for the TE-like waveguide mode are shown in Figure 2c. A broadband insertion loss of approximately $2 \mathrm{~dB}$ per facet is observed for the longer and higher bridges, i.e., the ones bridging gaps of $110 \mu \mathrm{m}$ and $170 \mu \mathrm{m}$ and a central height of $20 \mu \mathrm{m}$ and $30 \mu \mathrm{m}$, respectively. The high insertion loss for shorter bridges is assumed to be due to increasing non-adiabaticity of the planar-3D waveguide transition. For TM-polarized light, we observe a qualitatively similar trend of decreasing transmission with smaller bridge lengths. However, the insertion loss per facet is about $2 \mathrm{~dB}$ higher. Nevertheless, the overall transmission through the bridge is still sufficiently high to allow for efficient interconnection of arbitrary objects to on-chip planar waveguides. By comparing to the insertion loss measured for waveguides in contact with the substrate, we attribute the excess insertion loss to additional radiation loss occurring at the bend sections of the bridge.
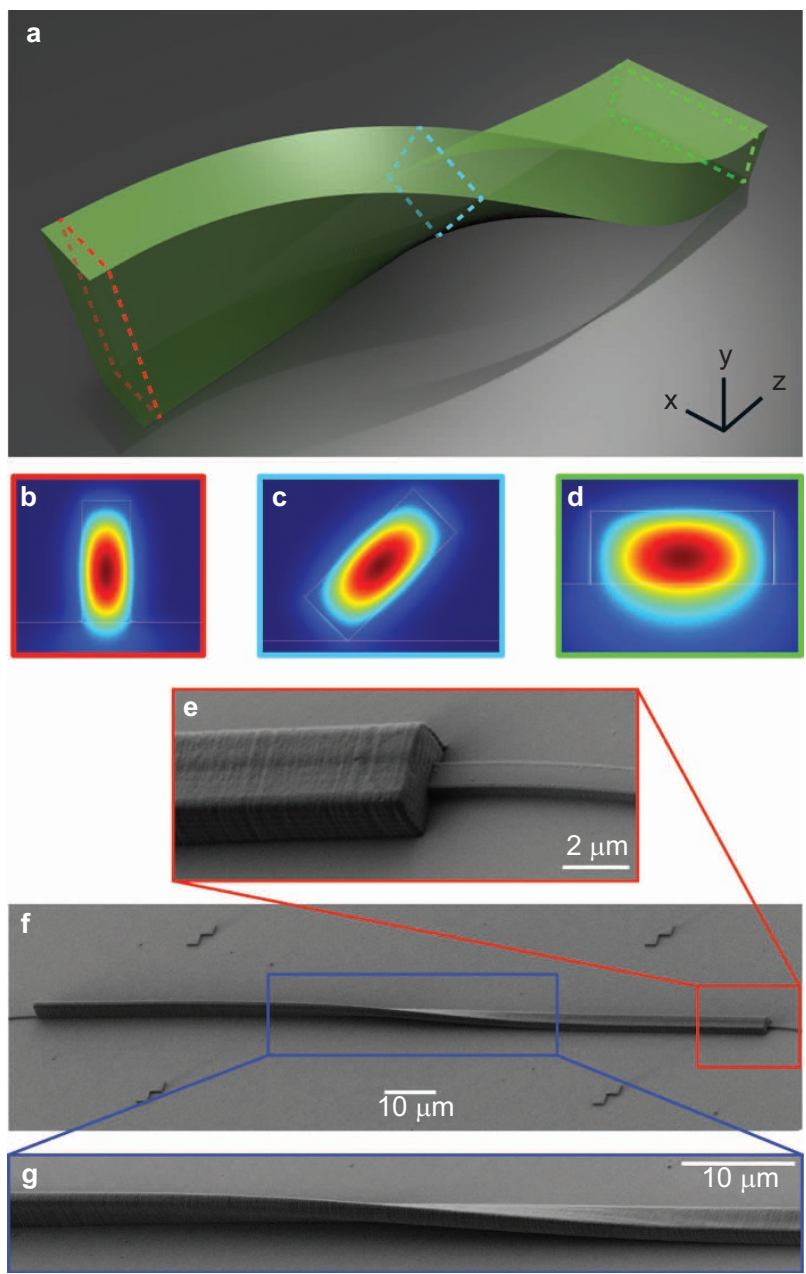

Figure 3 Broadband polarization conversion (a) Scheme showing the principle of operation of the mode evolution-based polarization rotator. As the cross-section of the rotator is twisted, the mode is expected to rotate as well. (b-d) COMSOL FEM simulations of electric field mode profiles at the positions marked in (a). (e-g) SEM images of the fabricated polarization rotator connecting two facing tapered nanophotonic waveguides. FEM, finite element method; SEM, scanning electron microscope. 


\section{Polarization rotators}

With access to 3D form shaping on a submicron-scale, waveguide geometries that cannot be achieved by traditional nanofabrication techniques can readily be produced. This additional degree of freedom is of particular interest for achieving control of the polarization of propagating optical modes on a chip, which is non-trivial with planar architectures.

Using 3D waveguides to achieve polarization rotation of a propagating mode, we employ DLW to twist the waveguide along the propagation direction. Such devices can be integrated into planar circuits using the polymer-inverted tapers described above. The twist, however, induces coupling among all waveguide modes. Thus, in order to optimize polarization rotation of a given mode, two properties of the rotator can be tuned. First, the number of guided modes should be ideally one and the propagation constants of the remaining modes should differ from each other as much as possible. The latter can be achieved by employing a rectangular waveguide with a large aspect ratio. Second, for a given cross-section of the waveguide, increasing the twist length will improve polarization rotation. ${ }^{31}$

We employ a twisted 3D rectangular waveguide fabricated by DLW as a polarization rotator. The design is again optimized by FDTD simulations for optimal conversion efficiency with minimal device length. The final design has a cross-section of $1.2 \times 3 \mu \mathrm{m}^{2}$ and is twisted along a length of $100 \mu \mathrm{m}$ (Figure 3). Eigenmode simulations using the finite-element simulation software COMSOL ${ }^{55}$ show that for this particular cross-section of the waveguide, the number of guided modes is minimal. Assuming refractive indices of 1.444 for the oxidized silicon layer and 1.54 for the exposed DLW resist, one TM-like mode $\left(n_{\mathrm{eff}}=1.450\right)$ is guided in the $3 \mu \mathrm{m}$ high end of the rotator. In the shallow end ( $1.2 \mu \mathrm{m}$ high), however, one TM-like $\left(n_{\text {eff }}=1.462\right)$ and one TE-like mode $\left(n_{\text {eff }}=1.468\right)$ are guided for a wavelength of $1550 \mathrm{~nm}$. Having found the eigenmodes with FEM simulations to illustrate the modal evolution along the polymer rotator, we then use FDTD simulations to quantitatively optimize the rotation performance by varying the geometry of the device. Even though propagation loss is difficult to predict accurately, relative comparison between simulated geometries allows for structural optimization. With the help of FDTD simulations, a minimum length of $80 \mu \mathrm{m}$ is found to be necessary for optimal performance of the rotator. For longer polarization rotators, the net rotation efficiency is predicted to be around $95 \%$.

In order to measure the rotation efficiency of the polarization rotator, we use nanophotonic circuits as shown in Figure $4 \mathrm{a}$ and $4 \mathrm{~b}$. The polymer waveguide section is written on our DLW set-up within a 20-min exposure. In these circuits, the laser light is coupled into the planar waveguides using port 2, where a TM grating coupler is located. Thus, mainly the TM-like waveguide mode is excited. After a Y-splitter, one half of the TM light is guided towards a reference TM grating coupler and is detected there. The reference coupler is used to calibrate the optical intensity in the waveguide, which is needed for determining the rotation efficiency of the device. The other half of the input TM light is guided through the polymer polarization rotator. After rotation, we couple the light out through a TE grating coupler, which is efficient for the TE-like mode. Similar to the design on the TM side, we employ also a Y-splitter and a second grating coupler optimized for $\mathrm{TE}$, to calibrate the rotated intensity in the output waveguide.

In addition, we repeat the experiment on the same device, but use port $3(\mathrm{TE})$ as input and port $2(\mathrm{TM})$ and port $4(\mathrm{TE})$ as outputs this time. Ignoring propagation losses in the planar waveguides, the following transmittances (relative to the laser output power) can be measured:

$$
\begin{aligned}
& T_{2 \rightarrow 1}=1 / 2 \alpha_{\mathrm{TM}}^{2} \\
& T_{2 \rightarrow 3}=1 / 2 \alpha_{\mathrm{TM}} \eta \gamma_{\mathrm{TE}} \alpha_{\mathrm{TE}} \\
& T_{3 \rightarrow 4}=1 / 2 \alpha_{\mathrm{TE}}^{2}
\end{aligned}
$$

Here, $\alpha_{\mathrm{TE}, \mathrm{TM}}$ is the coupling efficiency for the TE and TM couplers, respectively. $\eta$ is the rotation efficiency of the polarization rotator in the sense that this fraction of the TM-like light in the feeding waveguide towards the rotator is in the rotated (TE-like) state after passing the rotator and coupling back into the planar waveguide. The additional
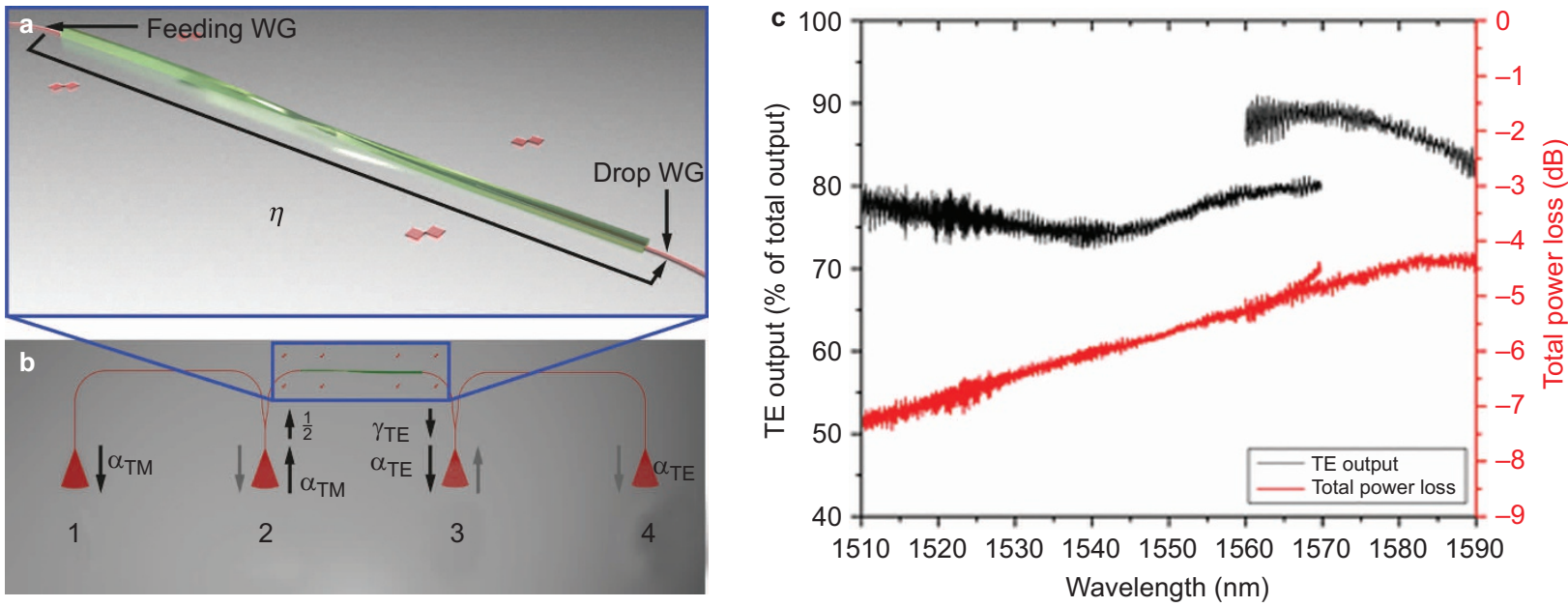

Figure 4 Measurement of polarization rotator (a) Close-up of the rotator between the feeding waveguide and the drop waveguide. $\eta$ is the rotation efficiency of the rotator. (b) Light is coupled into the chip on port 2, where a TM grating coupler excites the TM-like waveguide mode. One half of the light is guided towards reference port 1, the other half passes the polarization rotator. The TE-like portion of the light is efficiently coupled out through a TE grating coupler on port 3 . Port 4 is used as reference port in an inverted experiment, where initially the TE-like mode is excited through port 3. (c) Plot showing the results for the forward experiment, i.e., the relative power leaving the rotator in the rotated polarization state (black curve; normalized to the total power leaving the rotator), and the power loss induced by the polarization rotator (red curve). See text for details. TE, transverse electric; TM, transverse magnetic. 
factor $\gamma_{\mathrm{TE}}$ in the expression for $T_{2 \rightarrow 3}$ is due to the fact that the Y-splitter only transmits a fraction $\gamma_{\mathrm{TE}}$ of the incident TE-like mode when used in the backward direction. This factor is determined in a separate transmission measurement, but with a nanophotonic device on the same chip, such that the chip alignment relative to the fiber array is the same. In order to relate the fraction of the rotated light $\eta$ to the total output power of the polarization rotator, we measure the fraction of the light whose polarization is not rotated. Therefore, a similar nanophotonic circuit on the same chip is employed. Only the TE grating couplers on port 3 and port 4 are replaced by TM couplers there. For this circuit, the transmission from port 2 to port 3 relative to the laser output power becomes

$$
T_{2 \rightarrow 3}^{\prime}=1 / 2 \alpha_{\mathrm{TM}}^{\prime 2} \gamma_{\mathrm{TM}} t_{\mathrm{TM}}
$$

where $t_{\mathrm{TM}}$ is the transmittance for the TM-like mode through the polarization rotator and $\gamma_{\mathrm{TM}}$ is the fraction of a TM-like mode that is transmitted through the Y-splitter in backward direction. This can be measured in the same way as $\gamma_{\mathrm{TE}}$, but may have a different value. The coupling efficiency of the TM grating coupler in this nanophotonic circuit $\alpha_{\mathrm{TM}}^{\prime}$ is calculated separately from the transmission from port 2 to port 1 there $\left(T_{2 \rightarrow 1}^{\prime}\right)$, as it may be slightly different from the one obtained in the first circuit due to slightly different alignment of the fiber array. Finally, the ratio between the polarization-rotated light intensity (in the TE-like mode) and the total intensity in the drop waveguide (TElike+TM-like mode) after the rotator $q_{\mathrm{TE}}$ can be calculated:

$$
q_{\mathrm{TE}}=\frac{\eta}{t_{\mathrm{TM}}+\eta}=\frac{\gamma_{\mathrm{TM}} T_{2 \rightarrow 3} \sqrt{T_{2 \rightarrow 1}^{\prime}}}{\gamma_{\mathrm{TE}} T_{2 \rightarrow 3}^{\prime} \sqrt{T_{3 \rightarrow 4}}+\gamma_{\mathrm{TM}} T_{2 \rightarrow 3} \sqrt{T_{2 \rightarrow 1}^{\prime}}}
$$

This quantity can be understood as the net rotation efficiency of the polarization rotator.

For the rotator-induced relative power loss from feed to drop waveguide in units of $\mathrm{dB}$, we get

$$
L_{\mathrm{dB}}=10 \log \left(\eta+t_{\mathrm{TM}}\right)
$$

The measurement results for these two quantities for two exemplary polarization rotator devices are shown in Figure 4c. For these two rotators, the grating couplers have slightly different grating periods, such that their transmission profile and the suppression ratio are different as well. Because the grating couplers provide limited bandwidth, using two different sets of couplers allows us to characterize the performance of the rotator over a wider frequency range. From the measurements, we extract net rotation efficiency above $75 \%$ across the entire tuning range of our input laser. For the device with longer wavelength coverage, we measure maximum net rotation efficiency above $90 \%$, in close agreement to the FDTD simulations mentioned above (95\%). The discontinuity between the two measured devices is due to slightly different waveguide cross-sections, which vary between different DLW runs and thus, lead to slightly differing propagation properties.

We note that all calibration measurements rely on the fact that the suppression ratio between the coupling efficiency for TM and the coupling efficiency for TE for a given grating coupler (e.g., a TM grating coupler) is sufficiently large. Therefore, we measure the suppression prior to determining the rotation efficiency of the polarization rotator and show the efficiency and loss results only for the part of the spectrum where the suppression is better than $10 \mathrm{~dB}$. In order to obtain the polarization suppression spectrum for the grating couplers, we perform two pairs of subsequent transmission measurements. For the first set, we use a nanophotonic circuit comprising only two TE grating couplers connected through a planar waveguide. Prior to recording the transmission spectrum, we align the fiber array relative to the grating couplers. In the first measurement, we maximize transmission by adjusting the input polarization using the fiber polarization rotator. As TE grating couplers excite the TE waveguide mode efficiently, the transmission becomes

$$
T_{\mathrm{TE}}^{\mathrm{TE}}=\alpha_{\mathrm{TE}}^{\mathrm{TE}^{2}}
$$

where $T$ is the transmission relative to the laser output power and $\alpha$ is the coupling efficiency of the grating coupler. Subscripts denote the type of grating coupler (either TE or TM) and superscripts indicate the waveguide mode (also either TE or TM) that is coupled in or out of the chip by the grating. In the second step, we minimize transmission by only changing the input polarization. Then we get for the transmission through the nanophotonic circuit

$$
T_{\mathrm{TE}}^{\mathrm{TM}}=\alpha_{\mathrm{TE}}^{\mathrm{TM}^{2}}
$$

since now predominantly the TM mode is excited by the grating coupler. Finally, we define the suppression for the TE grating coupler $s_{\mathrm{TE}}^{\mathrm{dB}}$ as
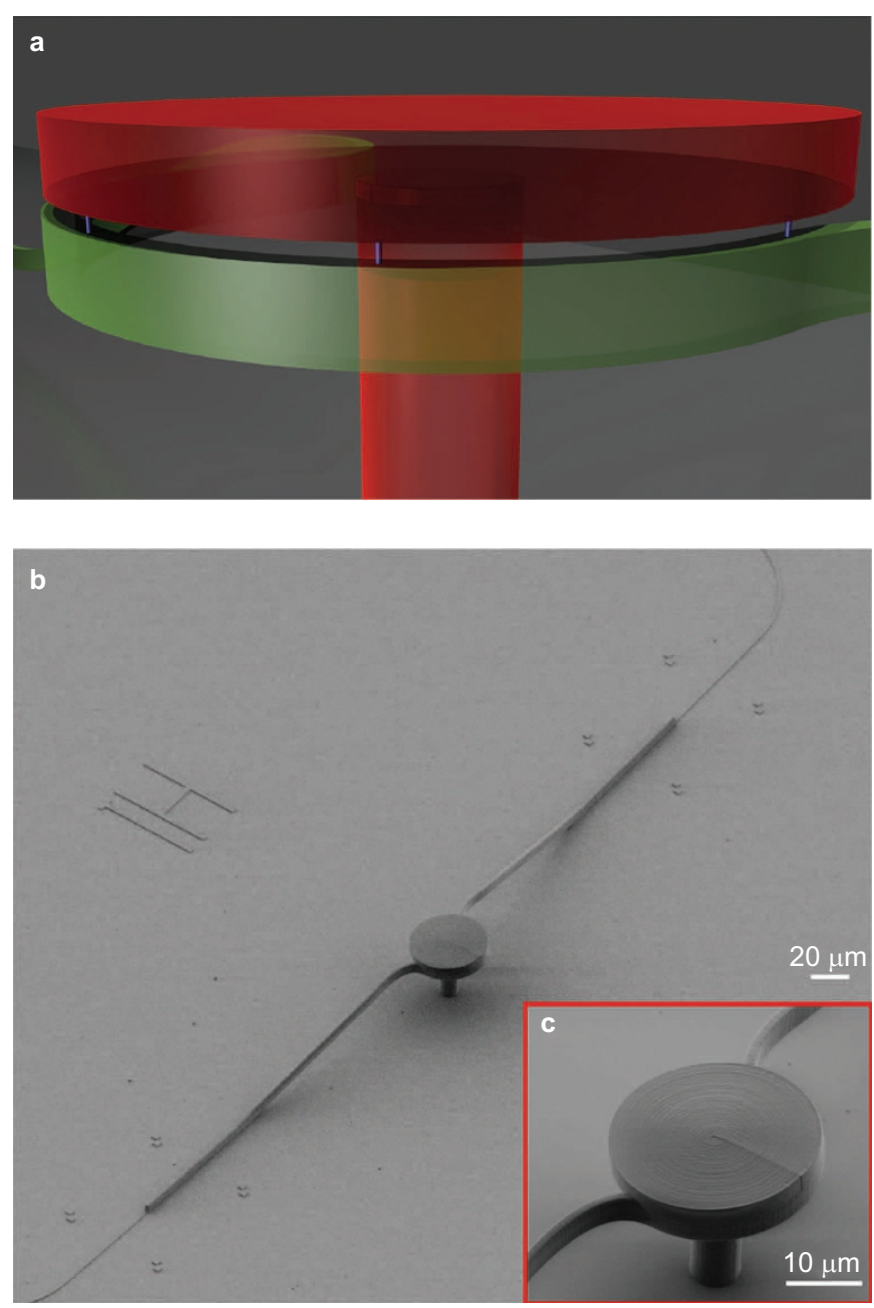

Figure 5 Disk resonator coupled to bridge waveguide. (a) Scheme of the bridge waveguide (green) written closely below the rim of the disk resonator (red). The gap is stabilized by spacers (blue), also fabricated by DLW. (b-c) SEM images of the fabricated bridge waveguide and disk resonator coupled to a nanophotonic silicon nitride circuit. DLW, direct laser writing; SEM, scanning electron microscope. 


$$
s_{\mathrm{TE}}^{\mathrm{dB}}=10 \log \left(\frac{\alpha_{\mathrm{TE}}^{\mathrm{TE}}}{\alpha_{\mathrm{TE}}^{\mathrm{TM}}}\right)
$$

To get the suppression for the TM grating coupler, an analogous measurement is carried out. Only the TE grating couplers are replaced by TM gratings and thus maximum transmission is obtained for the TM mode being excited by the coupler.

Experimentally we find that $s_{\mathrm{TE}}^{\mathrm{dB}}$ is well above $10 \mathrm{~dB}$ for the whole spectral band covered by our set-up. The $10 \mathrm{~dB}$ suppression bandwidth for TM grating couplers, however, is only $60 \mathrm{~nm}$. In order to cover the whole spectral band of our set-up, we thus employ two types of TM grating couplers with slightly different grating periods.

\section{Microdisk resonators}

Besides rotating the polarization of light, the ability to couple light into $3 \mathrm{D}$ devices can be exploited to evanescently couple to free-standing 3D resonators. As a demonstration, we fabricate both a 3D bridge waveguide and a free-standing disk resonator close to it (Figure 5a) using a
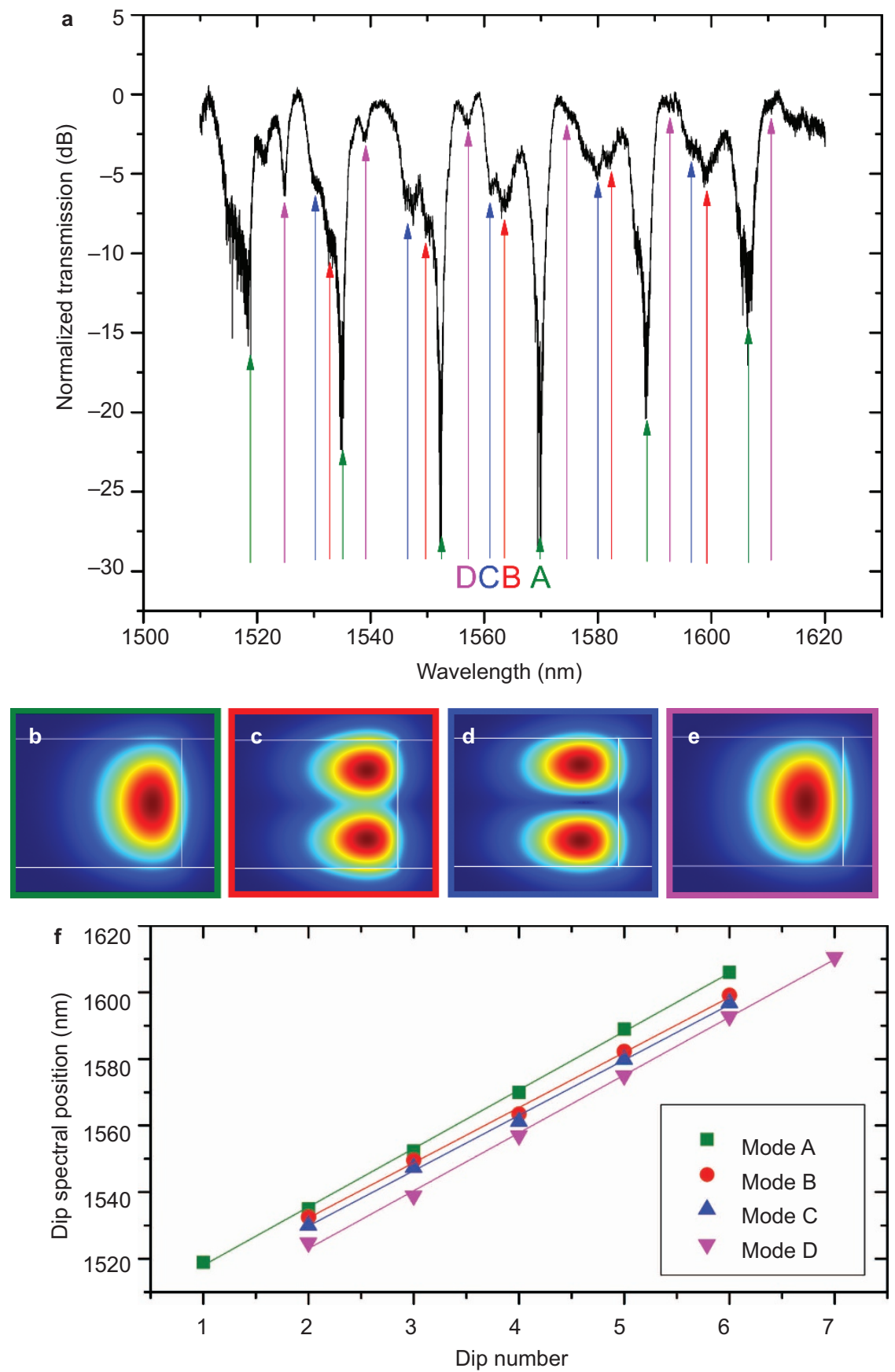

Figure 6 Resonator mode spectrum. (a) Transmission through bridge waveguide evanescently coupled to the disk resonator. Four sets of resonance dips can be identified (A-D). For mode A, we observe critical coupling, the quality factor of the resonator is around 1000. (b-e) COMSOL simulations of the mode profiles of the four modes identified in (a). (f) Plotting the spectral position of the resonance dips over the dip number, the free spectral range between individual dips is deduced from the slope of the linear regression. 
single DLW lithography step. The gap between resonator and waveguide is designed to be $500 \mathrm{~nm}$, but from scanning electron microscope (SEM) images (Figure $5 \mathrm{~b}$ and $5 \mathrm{c}$ ), we find that the structures attach to each other. The disk resonator is designed to have a diameter of $30 \mu \mathrm{m}$ and a nominal thickness of $2 \mu \mathrm{m}$, supported by a central pedestal with a diameter of $7 \mu \mathrm{m}$. The bridge waveguide is again coupled to a nanophotonic circuit. Here, however, the 3D waveguide has a height of $3 \mu \mathrm{m}$ and a width of $1.2 \mu \mathrm{m}$, such that it only guides a TM-like mode. These structures are realized in a DLW session of roughly $2 \mathrm{~h}$ per device. To couple to the TM mode, we do not use inverse tapers for the connection to the nanophotonic waveguide, i.e., the rectangular cross-section of $1.2 \times 3 \mu \mathrm{m}^{2}$ is maintained throughout the whole waveguide. Since for TM-like light, the evanescent field will be most prominent below and above the 3D bridge waveguide, we couple light from the waveguide into the disk in the vertical direction as illustrated in Figure 5a. In this way, the microdisk is written above the waveguide. In order to increase the coupling length, the trajectory of the bridge waveguide follows the rim of the disk for half a disk circumference.

We study the coupling between bridge waveguide and resonator by conducting the same type of transmission measurement as for the bridge waveguide characterization alone (see above). By adjusting the polarization of the incoming laser light, we predominantly launch TM-polarized light into the planar circuits. Nevertheless, because the guided mode is of a quasi-TM type, there is residual coupling to TE polarization in the planar waveguides as well in the free-standing polymer structures. In the transmission spectrum (Figure 6a), we identify four sets of resonance dips that are regularly spaced, corresponding to different resonator modes (Figure 6b-6e). The free spectral range (FSR) for the respective mode is obtained through a linear fit of the spectral positions of the dips as shown in Figure 6f. In order to assign the resonance dips to the corresponding whispering-gallery modes of the disk resonator, we simulate the eigenmodes of the disk resonator using COMSOL. ${ }^{56,57}$ The simulations are performed for a microdisk resonator without the waveguide present. Thus, the results predict the properties of the intrinsic microdisk, which can be analyzed experimentally in the limit of weak coupling. Nevertheless, by comparing the experimentally found resonance positions for different devices with varying coupling conditions, we find that the absolute value of the FSR does not significantly change when the waveguide is brought closer to the disk. By extracting the spectral difference between eigenfrequencies of successive azimuthal mode orders, we obtain the simulated FSR. Experimental and simulated FSRs are in good agreement and thus allow us to assign individual modes to the transmission dips within one FSR as shown in Figure 6b-6f. Here modes A and D are the $\mathrm{TM}$ and TE polarization of a first-order radial first-order axial mode, respectively, whereas $\mathrm{B}$ and $\mathrm{C}$ correspond to $\mathrm{TM}$ and TE polarization of a first-order radial second-order axial mode. We note that the resonance dips for the TE mode are significantly suppressed due to much weaker coupling to the predominantly TM-polarized waveguide mode. Because of residual coupling to the TE mode, however, both polarizations can be identified in the spectrum. The quality factor of the cavity is estimated to a value around 1000 by Lorentzian fits to the resonance dips. The comparatively low quality factor ${ }^{58}$ is attributed to the fact that bridge waveguide and disk resonator are touching and because of residual surface roughness of the resonator.

\section{CONCLUSIONS}

In this work, we demonstrate example applications of two-photon DLW for integrated optics. We combine the freedom to produce nearly arbitrary 3D polymer structures with the well-known fabrication and reliable measurement of quasiplanar silicon nitride devices. Along these lines, we are able to fabricate photonic devices that are difficult to obtain by conventional planar lithography techniques. First, we show efficient coupling of light from planar rib waveguides to a $3 \mathrm{D}$ bridge waveguide. Insertion loss is below $2 \mathrm{~dB}$ per facet at a wavelength of $1550 \mathrm{~nm}$. Second, we present a broadband mode evolution-based polarization rotator for the telecoms C-band with net conversion efficiencies exceeding $75 \%$ at a power loss of 5-7 dB. As a third example, we show coupling between a 3D bridge waveguide and a freestanding polymer disk resonator.

Further applications of interest that could prospectively be investigated include on-chip coupling to ultra-high $\mathrm{Q}$ resonators like microspheres $^{59}$ or convenient on-chip access to 3D photonic crystals. ${ }^{22,23}$ Also, the approach could be extended to the visible regime. For the silicon nitride devices, this is readily done by adjusting grating coupler period and waveguide geometry. For the polymer structures, however, resolution is limited by the DLW voxel size. Thus, in order to fabricate monomode waveguides for visible light, usage of more advanced 3D lithography techniques like stimulated-emission-depletion DLW $^{60}$ could be required.

\section{ACKNOWLEDGMENTS}

We would like to thank Christian Koos and Nicole Lindenmann at KIT for valuable discussions. We acknowledges support by DFG grant PE 1832/1-1 and PE 1832/2-1 and the Helmholtz Society through grant HIRG-0005. We also acknowledge support by the Deutsche Forschungsgemeinschaft (DFG) and the State of Baden-Württemberg through the DFG-Center for Functional Nanostructures (CFN) within subproject A1.04. We further want to thank Silvia Diewald and Stefan Kühn from the CFN Nanostructure Service Laboratory for assistance with the electron-beam exposition of the samples.

1 Shaw MJ, Guo J, Vawter GA, HabermehI S, Sullivan CT. Fabrication techniques for lowloss silicon nitride waveguides. Proc SPIE 2005; 5720: 109-118.

2 Lee $\mathrm{H}$, Chen T, Li J, Yang KY, Jeon S et al. Chemically etched ultrahigh-Q wedgeresonator on a silicon chip. Nat Photonics 2012; 6: 369-373.

3 Armani DK, Kippenberg TJ, Spillane SM, Vahala KJ. Ultra-high-Q toroid microcavity on a chip. Nature 2003; 421: 925-928.

4 Limon 0, Zalevsky Z. Nanophotonic interferometer realizing all-optical exclusive or gate on a silicon chip. Opt Eng 2009; 48: 064601.

5 Ohno F, Fukazawa T, Baba T. Mach-Zehnder interferometers composed of $\mu$-bends and $\mu$-branches in a Si photonic wire waveguide. Jpn J Appl Phys 2005; 44: 5322.

6 Zinoviev K, Carrascosa LG, del Río JS, Sepúlveda B, Domínguez C et al. Silicon photonic biosensors for lab-on-a-chip applications. Adv Opt Technol 2008; 2008 383927.

7 Camargo EA, Chong HM, de la Rue RM. Highly compact asymmetric Mach-Zehnder device based on channel guides in a two-dimensional photonic crystal. Appl Opt 2006; 45: 6507-6510.

8 Volkov VS, Bozhevolnyi SI, Devaux E, Laluet JY, Ebbesen TW. Wavelength selective nanophotonic components utilizing channel plasmon polaritons. Nano Lett 2007; 7: 880-884.

9 Hryniewicz JV, Absil PP, Little BE, Wilson RA, Ho PT. Higher order filter response in coupled microring resonators. IEEE Photonics Technol Lett 2000; 12: 320-322.

10 Djordjev K, Choi SJ, Choi SJ, Dapkus PD. Microdisk tunable resonant filters and switches. IEEE Photonics Technol Lett 2002; 14: 828-830.

11 Jones R, Doylend J, Ebrahimi P, Ayotte S, Raday 0 et al. Silicon photonic tunable optical dispersion compensator. Opt Express 2007; 15: 15836-15841.

12 Wong CW, Rakich PT, Johnson SG, Qi M, Smith HI et al. Strain-tunable silicon photonic band gap microcavities in optical waveguides. App/ Phys Lett 2004; 84: 1242-1244.

13 Okawachi Y, Foster M, Sharping J, Gaeta A, Xu Q et al. All-optical slow-light on a photonic chip. Opt Express 2006; 14: 2317-2322.

14 Yamada H, Chu T, Ishida S, Arakawa Y. Optical directional coupler based on Si-wire waveguides. IEEE Photonics Technol Lett 2005; 17: 585-587.

15 Stegmaier M, Pernice WHP. Broadband directional coupling in aluminum nitride nanophotonic circuits. Opt Express 2013; 21: 7304.

$16 \mathrm{McCoy} \mathrm{CH}$, Chen JT. Non-linear photonic switch and method of making the same. US Patent 20040046167, 2004. 
17 Liu X, Osgood RM, Vlasov YA, Green WM. Mid-infrared optical parametric amplifier using silicon nanophotonic waveguides. Nat Photonics 2010; 4: 557-560.

18 Foster MA, Turner AC, Sharping JE, Schmidt BS, Lipson M et al. Broad-band optical parametric gain on a silicon photonic chip. Nature 2006; 441: 960-963.

19 Smith BJ, Kundys D, Thomas-Peter N, Smith PG, Walmsley IA. Phase-controlled integrated photonic quantum circuits. Opt Express 2009; 17: 13516.

20 Peruzzo A, Laing A, Politi A, Rudolph T, O'Brien JL. Multimode quantum interference of photons in multiport integrated devices. Nat Commun 2011; 2: 224.

21 Thompson MG, Politi A, Matthews JC, O'Brien JL. Integrated waveguide circuits for optical quantum computing. IET Circuits Dev Syst 2011; 5: 94.

22 Noda S, Tomoda K, Yamamoto N, Chutinan A. Full three-dimensional photonic bandgap crystals at near-infrared wavelengths. Science 2000; 289: 604-606.

23 Lin SY, Fleming JG, Hetherington DL, Smith BK, Biswas R et al. A three-dimensional photonic crystal operating at infrared wavelengths. Nature 1998; 394: 251-253.

24 Xie Y, Lu Z, Li F, Zhao J, Weng Z. Lithographic fabrication of large diffractive optical elements on a concave lens surface. Opt Express 2002; 10: 1043-1047.

25 Verslegers L, Catrysse PB, Yu Z, White JS, Barnard ES et al. Planar lenses based on nanoscale slit arrays in a metallic film. Nano Lett 2009; 9: 235-238.

26 Jung YJ, Park D, Koo S, Yu S, Park N. Metal slit array Fresnel lens for wavelength-scale optical coupling to nanophotonic waveguides. Opt Express 2009; 17: 18852

27 Shackleford JA, Grote R, Currie M, Spanier JE, Nabet B. Integrated plasmonic lens photodetector. Appl Phys Lett 2009; 94: 083501.

28 Fu Y, Liu Y, Zhou X, Xu Z, Fang F. Experimental investigation of superfocusing of plasmonic lens with chirped circular nanoslits. Opt Express 2010; 18: 3438.

29 Li Z, Wu J, Shang L, Mickelson AR, Vachharajani M et al. A High-performance lowpower nanophotonic on-chip network. In: Proceedings of the 14th ACM/IEEE International Symposium on Low Power Electronics and Design. New York: ACM 2009, pp291-294.

30 Sherwood-Droz N, Lipson M. Scalable 3D dense integration of photonics on bulk silicon. Opt Express 2011; 19: 17758-17765.

31 Watts MR, Haus HA. Integrated mode-evolution-based polarization rotators. Opt Lett 2005; 30: 138 .

32 Feng J, Zhou Z. Polarization beam splitter using a binary blazed grating coupler. Opt Lett 2007; 32: 1662.

33 Melloni A, Morichetti F, Martinelli M. Polarization conversion in ring resonator phase shifters. Opt Lett 2004; 29: 2785 .

34 Shani Y, Henry CH, Kistler RC, Kazarinov RF, Orlowsky KJ. Integrated optic adiabatic polarization splitter on silicon. App/ Phys Lett 1990; 56: 120.

35 Ding $\mathrm{YH}$, Ou HY, Peucheret C. Wideband polarization splitter and rotator with large fabrication tolerance and simple fabrication process. Opt Lett 2013; 38: 1227-1229.

36 Zhang J, Yu M, Lo GQ, Kwong DL. Silicon-waveguide-based mode evolution polarization rotator. IEEE J Sel Top Quantum Electron 2010; 16: 53-60.

37 Roels J, de Vlaminck I, Lagae L, Maes B, van Thourhout D et al. Tunable optical forces between nanophotonic waveguides. Nat Nanotechnol 2009; 4: 510-513.

38 Li M, Pernice WH, Tang HX. Tunable bipolar optical interactions between guided lightwaves. Nat Photonics 2009; 3: 464-468.

39 Ollier E, Philippe P, Chabrol C, Mottier P. Micro-opto-mechanical vibration sensor integrated on silicon. J Light Technol 1999; 17: 26.

40 Ollier E, Labeye P, Mottier PL. Micro-optomechanical switch for optical fiber networks. In: Armenise MN, Wong KK, editors. Functional Photonic Integrated Circuits. Bellingham, WA: International Society for Optics and Photonics; 1995. pp116-124.

41 Rath P, Khasminskaya S, Nebel C, Wild C, Pernice WH. Diamond-integrated optomechanical circuits. Nat Commun 2013; 4: 1690.

42 Kawata S, Sun HB, Tanaka T, Takada K. Finer features for functional microdevices. Nature 2001; 412: 697-698.
43 von Freymann G, Ledermann A, Thiel M, Staude I, Essig S et al. Three-dimensional nanostructures for photonics. Adv Funct Mater 2010; 20: 1038-1052.

44 Deubel M, von Freymann G, Wegener M, Pereira S, Busch K et al. Direct laser writing of three-dimensional photonic-crystal templates for telecommunications. Nat Mater 2004; 3: 444-447.

45 Bückmann T, Stenger N, Kadic M, Kaschke J, Frölich A et al. Tailored 3D mechanical metamaterials made by dip-in direct-laser-writing optical lithography. Adv Mater 2012; 24: 2710-2714.

46 Zhang YL, Chen QD, Xia H, Sun HB. Designable 3D nanofabrication by femtosecond laser direct writing. Nano Today 2010; 5: 435-448.

47 Bueckmann T, Schittny R, Thiel M, Kadic M, Milton GW et al. On three-dimensional dilational elastic metamaterials. New J Phys 2014; 16 033032: doi: 10.1088/13672630/16/3/033032.

48 Lindenmann N, Balthasar G, Hillerkuss D, Schmogrow R, Jordan M et al. Photonic wire bonding: a novel concept for chip-scale interconnects. Opt Express 2012; 20 17667-17677.

49 Lindenmann N, Balthasar G, Jordan M, Hillerkuss D, Schmogrow R et al. Low-loss photonic wire bond interconnects enabling $5 \mathrm{Tbit} / \mathrm{s}$ data transmission. In: Proceedings of Optical Fiber Communication Conference; 4-8 March 2012; Los Angeles, CA, USA. IEEE: Washington, DC, USA, 2012, pp1-3.

50 Maire G, Vivien L, Sattler G, Kazmierczak A, Sanchez B et al. High efficiency silicon nitride surface grating couplers. Opt Express 2008; 16: 328.

51 Doerr CR, Chen L, Chen YK, Buhl LL. Wide bandwidth silicon nitride grating coupler. IEEE Photonics Technol Lett 2010; 22: 1461-1463.

52 Taillaert D, van Laere F, Ayre M, Bogaerts W, van Thourhout D et al. Grating couplers for coupling between optical fibers and nanophotonic waveguides. Jpn J Appl Phys 2006; 45: 6071-6077.

53 Taflove A. Computational Electrodynamics: the Finite-Difference Time-Domain Method. 3rd ed. Boston, MA: Artech House; 2005.

54 Oskooi AF, Roundy D, Ibanescu M, Bermel P, Joannopoulos JD et al. Meep: a flexible free-software package for electromagnetic simulations by the FDTD method. Comput Phys Commun 2010; 181: 687-702.

55 COMSOL Multiphysics®. Stockholm: COMSOL, Inc.; 2011. http://www.comsol.com/ comsol-multiphysics

56 Oxborrow M. Traceable 2D finite-element simulation of the whispering-gallery modes of axisymmetric electromagnetic resonators. IEEE Trans Microw Theory Tech 2007; 55: $1209-1218$

57 Oxborrow M. How to simulate the whispering-gallery modes of dielectric microresonators in FEMLAB/COMSOL. Proc SPIE 2007; 6452: 64520J.

58 Schell AW, Kaschke J, Fischer J, Henze R, Wolters J et al. Three-dimensional quantum photonic elements based on single nitrogen vacancy-centres in laser-written microstructures. Sci Rep 2013; 3: 1577.

59 Collot L, Lefèvre-Seguin V, Brune M, Raimond JM, Haroche S. Very high-Q whisperinggallery mode resonances observed on fused silica microspheres. EPL Europhys Lett 1993; 23: 327.

60 Fischer J, Wegener M. Three-dimensional direct laser writing inspired by stimulatedemission-depletion microscopy. Opt Mater Express 2011; 1: 614.

\section{(c) (1) (2) This work is licensed under a Creative Commons Attribution-}

(c) ${ }_{\text {BY }}$ NC SA NonCommercial-ShareAlike 3.0 Unported License. The images or other third party material in this article are included in the article's Creative Commons license, unless indicated otherwise in the credit line; if the material is not included under the Creative Commons license, users will need to obtain permission from the license holder to reproduce the material. To view a copy of this license, visit http://creativecommons.org/licenses/by-nc-sa/3.0/ 